\title{
CircNRIPI Modulates the miR-5I5-5p/lL-25 Axis to Control 5-Fu and Cisplatin Resistance in Nasopharyngeal Carcinoma
}

This article was published in the following Dove Press journal: Drug Design, Development and Therapy

\author{
Junwu $\operatorname{Lin}^{1}, *$ \\ Hong Qin $^{2} * *$ \\ Yue $\mathrm{Han}^{3}$ \\ Xinghua $\mathrm{Li}^{4}$ \\ YuJuan Zhao ${ }^{5}$ \\ Guangsheng Zhai $\mathbb{D D}^{6}$ \\ 'Department of Otolaryngology, Central \\ Hospital, Qingdao, Shandong, People's \\ Republic of China; ${ }^{2}$ Department of \\ Otolaryngology, Women and Children's \\ Hospital, Qingdao, Shandong, People's \\ Republic of China; ${ }^{3}$ Department of \\ Urology, Zhangqiu District People's \\ Hospital, Jinan, Shandong, People's \\ Republic of China; ${ }^{4}$ Department of \\ Critical Care Medicine, Zhangqiu District \\ People's Hospital, Jinan, Shandong, \\ People's Republic of China; ${ }^{5}$ Department \\ of Otolaryngology, Rizhao Second \\ People's Hospital, Rizhao, Shandong, \\ People's Republic of China; ${ }^{6}$ Department \\ of Radiotherapy, Zibo Central Hospital, \\ Zibo, Shandong, People's Republic of \\ China
}

*These authors contributed equally to this work
Background: The development of drug resistance leads many NPC patients to experience disease relapse following the completion of chemotherapy. It is thus essential that the mechanistic basis for such chemoresistance be clarified in an effort to identify approaches to sensitizing NPC tumors to treatment with cisplatin and related agents.

Methods: A qRT-PCR approach was used to measure the expression of circNRIP1 in NPC, while luciferase assays were used to identify interactions with downstream targets of circNRIP1 activity including miR-515-5p and IL-25. CCK8 assays were also utilized to detect IC50 values for cisplatin and 5-Fu.

Results: The expression of circNRIP1 was significantly increased in the serum of chemoresistant NPC patients. At a functional level, we determined that circNRIP1 is able to sequester miR-515-5p, thereby inhibiting its ability to post-transcriptionally suppress IL-25 expression. We observed a significant negative correlation between the expression of miR-515-5p and circNRIP1 in serum samples from chemoresistant NPC patients, consistent with a functional interaction between these two factors. We further found that 5-Fu and CDDP IC50 values in NPC cells in which circNRIP1 had been knocked down were restored following miR-515-5p inhibitor transfection. Similarly, changes in these IC50 values were reversed in NPC cells transfected with miR-515-5p mimics following the overexpression of IL-25 in these same cells. Conclusion: These data highlight the circNRIP1/miR-515-5p/IL-25 as a novel regulator of $5-\mathrm{Fu}$ and cisplatin resistance in NPC, suggesting that this pathway may be amenable to therapeutic targeting as an approach to treating this cancer type.

Keywords: circNRIP1, 5-Fu resistance, cisplatin resistance, NPC, miR-515-5p, IL-25

\section{Introduction}

Nasopharyngeal carcinoma (NPC) is among the most common and deadliest forms of head and neck cancer globally, ${ }^{1,2}$ and while many improvements in the surgical and neoadjuvant chemotherapy-based treatment of this disease have been made in recent years, NPC patients have a poor prognosis with a 5-year survival rate of $50-70 \%{ }^{3,4}$ Cisplatin (CDDP) is currently the first-line treatment extended to NPC patients, but the development of cisplatin resistance and consequent tumor recurrence is common. It is thus essential that the mechanistic basis for cisplatin resistance in NPC tumors be better clarified in order to establish how best to sensitize these tumors to chemotherapeutic intervention.

Circular RNAs (circRNAs) are highly stable RNAs that form closed covalent loops without any 5'-cap or 3'-poly-A tail. ${ }^{5-9}$ The expression of circRNAs is evident in
Correspondence: Guangsheng Zhai Department of Radiotherapy, Zibo Central Hospital, No. 54, Communist Youth League West Road, Zhangdian District, Zibo 255036, Shandong, People's Republic of China Fax +86-18678I86986

Email guangshengzhai86@163.com 
many different cancer cell types including gastric cancer, ${ }^{10}$ colorectal cancer, ${ }^{11}$ lung adenocarcinoma, ${ }^{12}$ breast cancer, ${ }^{13}$ oral squamous cell carcinoma, ${ }^{14}$ hepatocellular carcinoma ${ }^{15}$ and $\mathrm{NPC}^{16}$ cells. Importantly, these circRNAs can strongly influence tumor cell function and differentiation by sequestering microRNAs (miRNAs) in a sequence-specific manner and thereby altering their regulatory activity. ${ }^{13}$

CircNRIP1 (hsa_circ_0004771) is a circRNA derived from the NRIP1 locus (chr21:16,386,664-16,415,895). Multiple studies have identified circNRIP1 as a driver of tumor progression. In breast cancer cells, silencing this circRNA inhibits tumor cell proliferation and promotes apoptotic cell death owing to consequent increases in miR-653 activity and resultant suppression of the ZEB2 signaling pathway. ${ }^{17}$ In cervical cancer, circNRIP1 has similarly been shown to sequester miR-629-3p and to thereby modulate the PTP4A1/ERK/1/2 pathway in order to promote tumor invasion and migration. ${ }^{18}$ Through comparable mechanisms, this circRNA also binds and sequesters miR-149-5p in gastric cancer cells and thereby promotes tumor progression via the AKT1/mTOR pathway. ${ }^{19}$ The specific functional relevance of circNRIP1 as a regulator of cisplatin resistance, however, has yet to be defined.

Herein, we determined that the expression of circNRIP1 was significantly elevated in the serum of cisplatin-resistant NPC patients, and we found that circNRIP1 downregulation was sufficient to reduce NPC cell resistance to cisplatin at least in part via modulating the miR-515-5p/IL-25 axis.

\section{Materials and Methods}

\section{Ethical Approval}

Samples of NPC patient serum were obtained from Zibo Central Hospital. The Ethics Committee of Zibo Central Hospital approved the present study, and all patients provided written informed consent to participate. The research was performed in accordance with the Declaration Helsinki principles.

\section{Cell Culture}

HK-1 (from the China Center for Type Culture Collection, Wuhan, China) and CNE-1 (from the Chinese Academy of Sciences, Shanghai, China) cells were grown in RPMI1640 (Gibco) containing 10\% fetal bovine serum (FBS) at $37^{\circ} \mathrm{C}$ in a humidified $5 \% \mathrm{CO}_{2}$ incubator. The cisplatinresistant HK-1 and CNE-1 cell line (HK-1/CDDP and
CNE-1/CDDP) was established by treating HK-1 and CNE-1 with gradient increased CDDP for 6 months.

\section{qRT-PCR}

Trizol (Invitrogen) was used to isolate total RNA from cells, after which a PrimeScript RT reagent kit (Takara Bio, Shiga, Japan) was used to prepare cDNA. Next, qRT-PCR reactions were conducted with a 7500 Real-time PCR System (Applied Biosystems, CA, USA) and a SYBR Green PCR Kit (Takara). The $2^{-\Delta \Delta \mathrm{Ct}}$ method was used to assess relative gene expression, and the following primers were used for this study: hsa_circ_0004771: forward 5'-TCCGGATGACATCAGAGC TAC-3' and reverse 5'-TCAAGTGTGCATCTTCTGGCT-3'; GAPDH: forward 5'-GCACCGTCAAGCTGAGAAC-3' and reverse 5'-TGGTGAAGACGCCAGTGGA-3'.

\section{CCK8 Assay}

Half maximal inhibitory concentration (IC50) values for 5-FU and cisplatin were assessed via Cell Counting Kit-8 (CCK-8, Dojindo, Japan) based on provided directions, with absorbance values at $450 \mathrm{~nm}$ being analyzed with a SpectraMax 250 instrument (Molecular Devices, USA).

\section{Luciferase Reporter Assay}

The starBase 3.0 was used for the prediction of the binding sites between CircNRIP1 or IL-25 and miR-515-5p. Subsequently, the sequence of wild type (WT) or mutant (MUT) CircNRIP1 or the 3'Untranslated Region (UTR) fragment of IL-25 harboring miR-515-5p binding sites was amplified and inserted into pGL3-control vector (Promega) for the construction of the luciferase reporters. WT or mutant luciferase reporter assay constructs were transfected into NPC cells using Lipofectamine 3000, together with miR-149 mimic constructs as appropriate. A Dual Luciferase Reporter System Kit (E1910, Promega, USA) was then used based on provided directions to analyze luciferase activity.

\section{ELISA}

Levels of IL-25 were analyzed with an ELISA kit (Abcam, MA, USA) based on provided directions.

\section{Statistics}

All experiments were repeated in triplicate, and data are given as means \pm standard deviation. Data were compared via Student's t-tests or ANOVAs, with $\mathrm{P}<0.05$ as the significance threshold. 


\section{Results}

\section{Chemoresistant NPC Patients Exhibit Elevated Serum circNRIPI Levels}

In this study, NPC patients that had undergone four cycles of combined 5-Fu/cisplatin treatment were classified into either chemoresistant $(n=72)$ or chemosensitive $(n=66)$ groups based upon the Huvos scoring system. ${ }^{20}$ Prior to treatment, serum circNRIP1 levels were comparable between these two patient groups, whereas after treatment the expression of this circNRIP1 was significantly elevated in serum samples from chemoresistant patients relative to those from chemosensitive patients (Figure 1A). Log-rank (Mantel-Cox) tests revealed that chemoresistant NPC patients with high serum circNRIP1 levels had a shorter overall survival duration relative to chemosensitive patients with low serum circNRIP1 levels (Figure 1B).

\section{CircNRIPI Knockdown Partially Reverses NPC Cell 5-Fu and Cisplatin Resistance}

We next evaluated the expression of circNRIP1 in cisplatin-resistant HK-1 and CNE-1 cells (HK-1/CDDP and CNE-1/CDDP cell), revealing that this circRNA was significantly upregulated in cisplatin-sensitive $\mathrm{HK}-1$ and CNE-1 cells (Figure 2A). We successfully utilized siRNA constructs to knock down circNRIP1 in HK-1/ CDDP and CNE-1/CDDP cells (Figure 2B). A CCK8 assay also demonstrated that circNRIP1 knockdown decreased the IC50 of 5-Fu (Figure 2C) and cisplatin (Figure 2D) in HK-1/CDDP and CNE-1/CDDP cells, indicating that the loss of this circRNA is sufficient to partially reverse the in vitro resistance of NPC cells to these two chemotherapeutic agents.

\section{CircNRIPI Functions by Sequestering miR-5I5-5p}

In order to understand the mechanisms whereby circNRIP1 controls NPC cell chemoresistance, we next leveraged the CircNet, RNA22, and RegRNA tools to identify miRNAs capable of binding to this circRNA, leading to the identification of miR-515-5p as a circNRIP1 target miRNA (Figure 3A). Luciferase reporter assays confirmed this regulatory relationship, as miR515-5p mimic transfection was sufficient to reduce the activity of WT but not mutant (MUT) luciferase reporter constructs (Figure 3B). We further found that circNRIP1 knockdown markedly increased miR-515-5p expression in HK-1/CDDP and CNE-1/CDDP cells (Figure 3C), and serum miR-515-5p levels were significantly lower in chemoresistant NPC patients relative to chemosensitive patients after treatment (Figure 3D). Importantly, serum circNRIP1 and miR-515-5p levels were negatively correlated with one another in chemoresistant patients (Figure 3E). The IC50 values for 5-Fu (Figure 3F) and CDDP (Figure 3G) were also restored in HK-1/CDDP and CNE-1/CDDP cells in which circNRIP1 had been knocked down when these cells were transfected with a miR-515$5 \mathrm{p}$ inhibitor.

\section{CircNRIPI Augments 5-Fu and Cisplatin Resistance in NPC Cells via Sequestering miR-5 I5-5p and Thereby Promoting IL-25 Expression}

In an effort to identify miR-515-5p targets within NPC cells, we next leveraged the TargetScan database, which revealed IL-25 as one such target gene (Figure 4A).
A

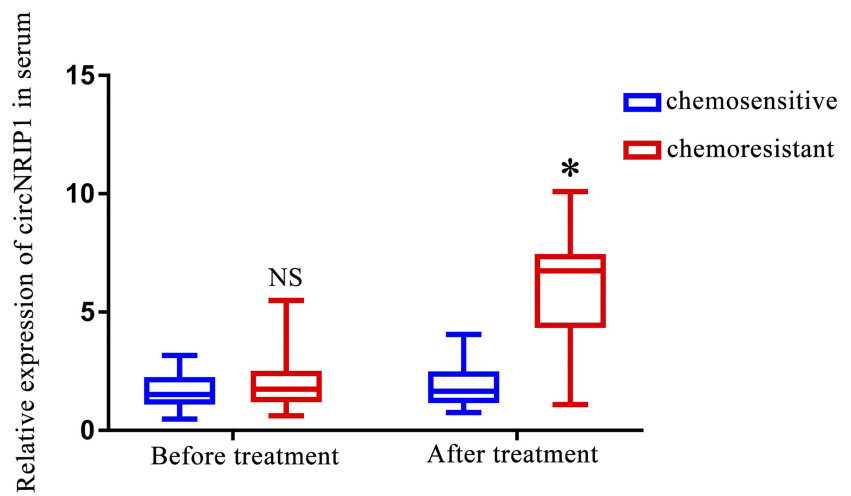

B

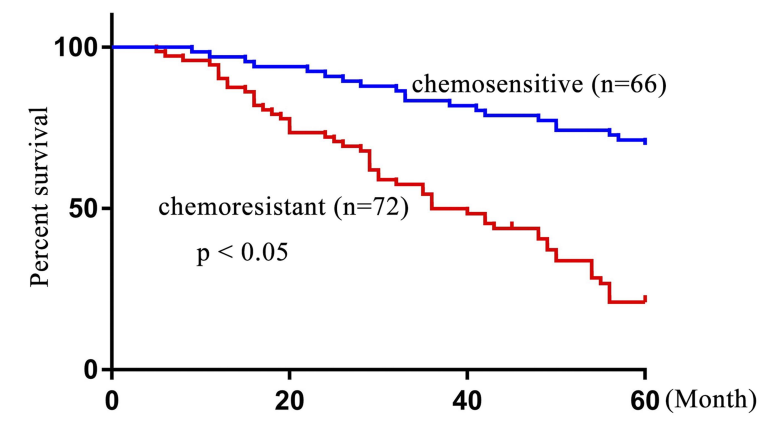

Figure I Levels of circNRIPI are increased in the serum of chemoresistant NPC patients. (A). circNRPI expression was assessed via qRT-PCR in chemoresistant and chemosensitive NPC patient serum samples. (B). The overall survival of chemoresistant patients was significantly lower than that of chemosensitive patients. $*_{P}<0.05$. 
A

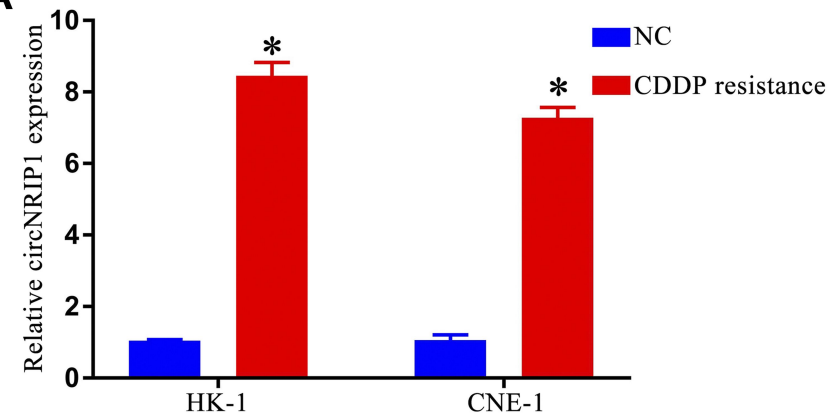

C

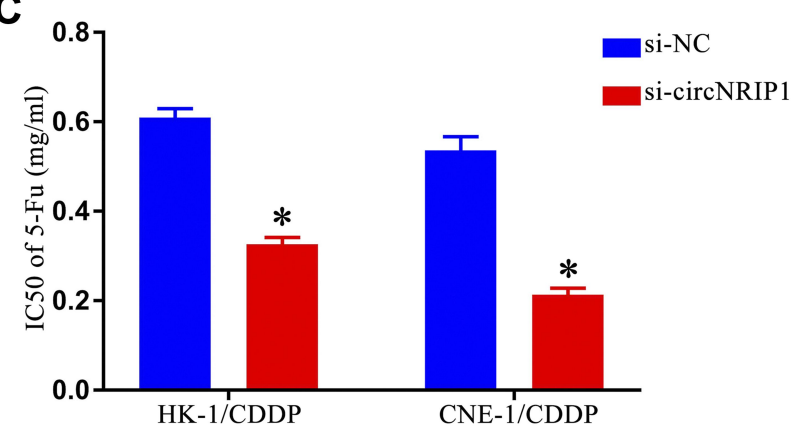

B

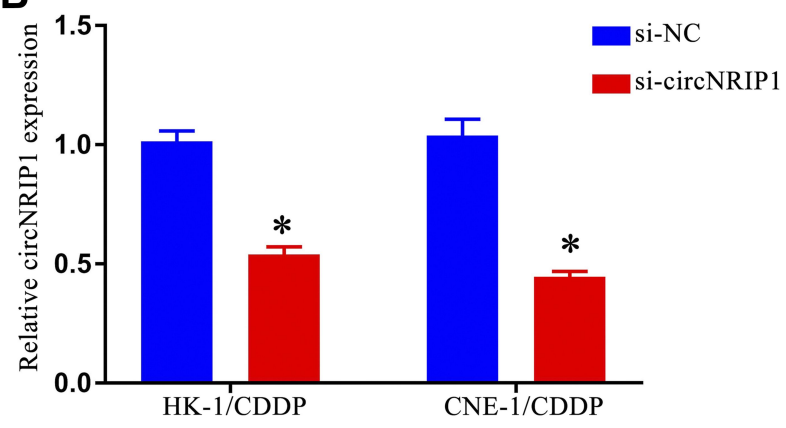

D

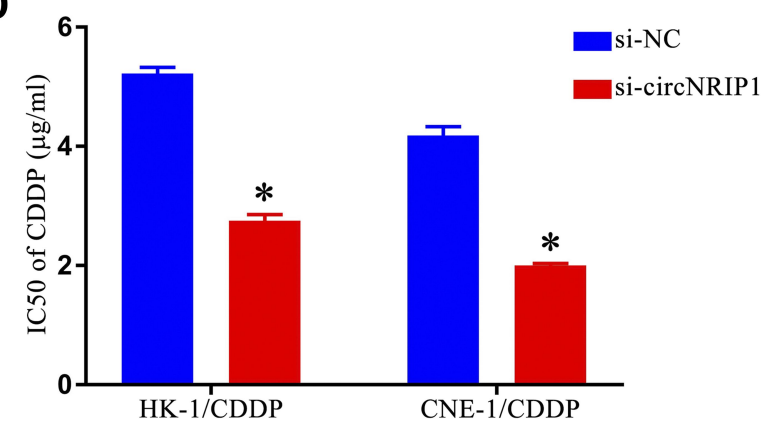

Figure 2 Knockdown of circNRIPI increased the sensitivity of NPC cells to 5-Fu and CDDP in vitro. (A). Expression of circNRIPI was significantly increased in HK-I/ CDDP and CNE-I/CDDP cells relative to HK-I and CNE-I cells (Figure 2A). (B). siRNA constructs were successfully used to knock down circNRIPI in HK-I/CDDP and CNE-I/CDDP cells, and circNRIPI knockdown decreased the IC50 values of 5-Fu (C) and CDDP (D) in HK-I/CDDP and CNE-I/CDDP cells. *P $<0.05$.

Luciferase reporter assays further confirmed that miR-515$5 p$ mimics were sufficient to decrease the activity of WT but not mutant IL-25 3'-UTR luciferase reporter constructs, confirming that IL-25 is a direct miR-515-5p target (Figure 4B). We determined that miR-515-5p overexpression suppressed IL-25 expression in HK-1/CDDP and CNE-1/CDDP cells, and transfection with IL-25 was sufficient to restore the expression of this cytokine in these cells (Figure 4C and D). Overexpressing miR-515-5p markedly reduced cisplatin and 5-Fu IC50 values in HK1/CDDP and CNE-1/CDDP cells, while IL-25 transfection was sufficient to reverse this phenotype (Figure 4E and F). As such, our data demonstrate that circNRIP1 enhances 5-Fu and cisplatin resistance in NPC cells at least in part via sequestering miR-515-5p and thereby promoting IL-25 expression.

\section{Discussion}

Herein, we found that circNRIP1 levels were significantly elevated in the serum of chemoresistant NPC patients relative to chemosensitive patients. We additionally determined that circNRIP1 functions by sequestering miR-515-5p and thereby inhibiting its ability to post-transcriptionally regulate the expression of IL-25 via binding to the IL-25 3'-
UTR. We detected a significant negative correlation between the expression of miR-515-5p and circNRIP1 in the serum of chemoresistant NPC patients, consistent with a functional relationship between these two RNAs. We further found that the knockdown of circNRIP1 significantly increased the sensitivity of cisplatin-resistant NPC cells to $5-\mathrm{Fu}$ and cisplatin, while miR-515-5p inhibitor transfection reversed this effect. In addition, the transfection of IL-25 into cells transfected with a miR-515-5p mimic was sufficient to restore 5 - $\mathrm{Fu}$ and cisplatin resistance. Together, these data suggest that the circNRIP1/miR-515$5 \mathrm{p} / \mathrm{IL}-25$ regulatory axis is a novel regulator of cisplatin and 5-Fu resistance in NPC cells. As such, therapeutic efforts to target this pathway may facilitate improved NPC treatment.

Many prior studies have shown that circRNAs function via sequestering target miRNAs in a sequence-specific manner, thereby reducing miRNA activity and increasing the expression of miRNA target genes. ${ }^{21-23}$ Certain circRNAs such as circRNA ZNF609, hsa_circ_0046263, and circ_0008450 have been shown to drive NPC progression through the competitive binding of endogenous miR-150-5p, miR-133a-5p, and miR-577, respectively. ${ }^{24-26}$ These results are consistent with our data suggesting that circNRIP1 functions as a competing endogenous RNA (ceRNA) specific for 
A

circNRIP1 WT 5'-GCAGAGGCUCAGAGCUUGGAGAC-3'

|||| $\mid$

miR-515-5p 3'-GUCUUUCACGAAAGAAAACCUCUU-5'

circNRIP1 MUT 5'-GCAGAGGCUCAGAGCUACCUCUC-3'

C

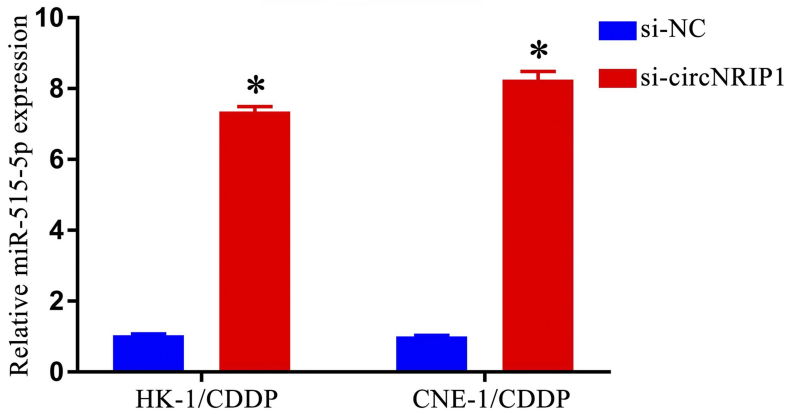

E

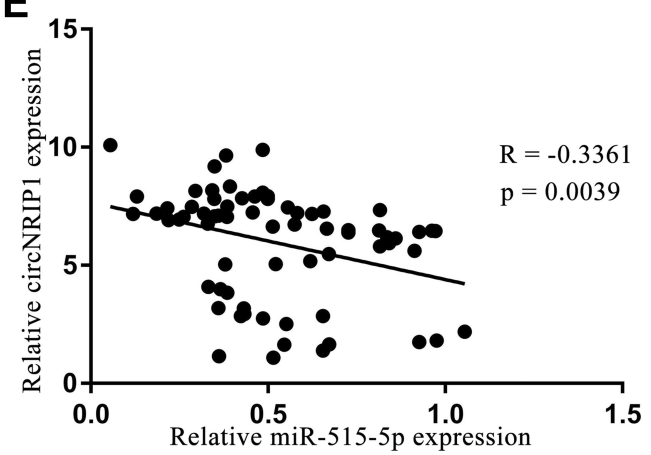

B

D
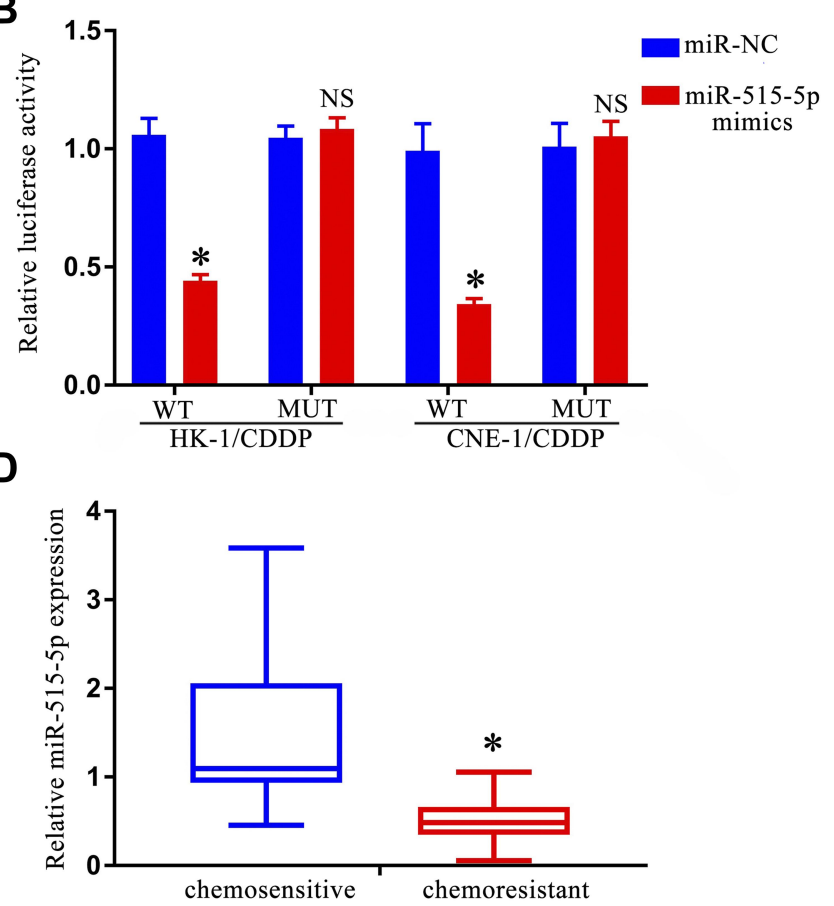

$\mathbf{F}$

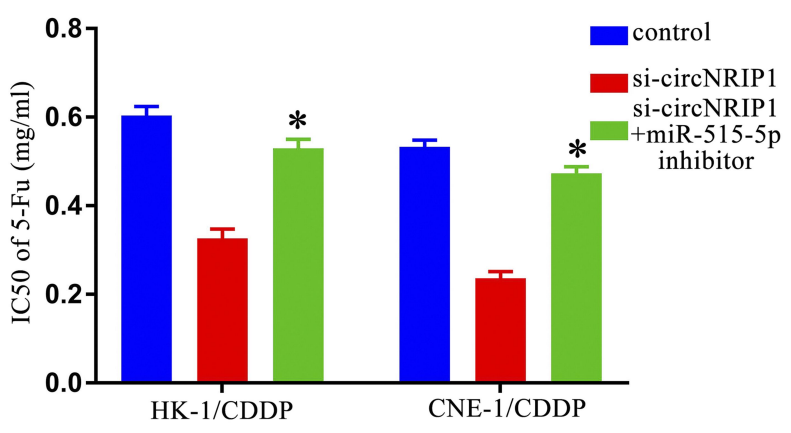

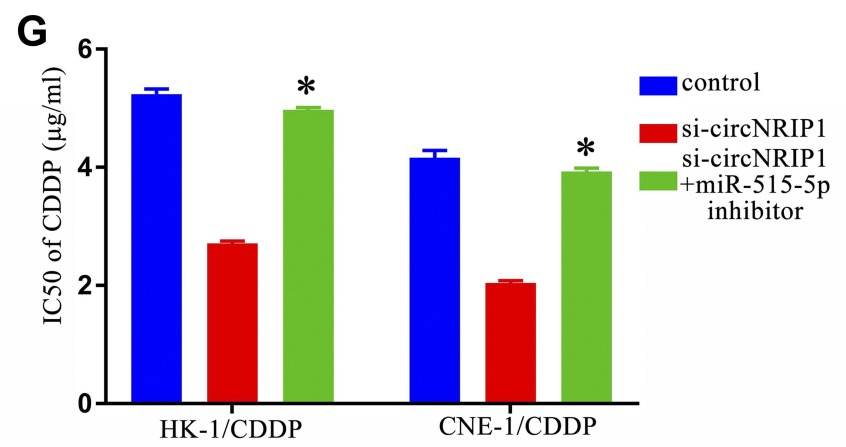

Figure 3 CircNRIPI functions by sequestering miR-5I5-5p. (A). Putative miR-5I5-5p binding sites within circNRIPI. (B). Transfection with miR-5I5-5p mimics was sufficient to reduce WT but not MUT luciferase reporter activity. (C). CircNRIPI knockdown markedly increased miR-5I5-5P expression in HK-I/CDDP and CNE-I/CDDP cells. (D). Following treatment, serum miR-5I5-5p levels were lower in the serum of chemoresistant NPC patients relative to chemosensitive patients. (E). Serum circNRIPI and miR-515-5p levels were negatively correlated in the serum of chemoresistant NPC patients. The IC50 value for 5-Fu (F) and CDDP (G) was restored in HK-I/CDDP and CNE-I/CDDP cells transfected with si-circNRIPI following miR-515-5p inhibitor transfection. ${ }^{N S}>0.05,{ }_{p}<0.05$.

miR-515-5p in NPC cells, thereby suppressing miR-515-5p expression and enhancing 5 -Fu and cisplatin resistance in these cells. These results are consistent with prior reports showing that miR-515-5p can inhibit tumor cell migration and metastasis, ${ }^{27}$ and with data indicating that miR-515-5p functions as a tumor suppressor via targeting TRIP13 in 
A

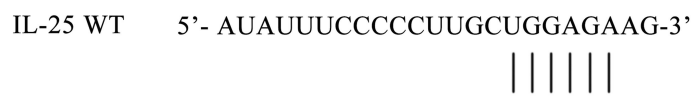

miR-515-5p 3'-GUCUUUCACGAAAGAAAACCUCUU-5'

IL-25 MUT 5'-AUAUUUCCCCCUUGCACCUCUAG-3'

\section{C}

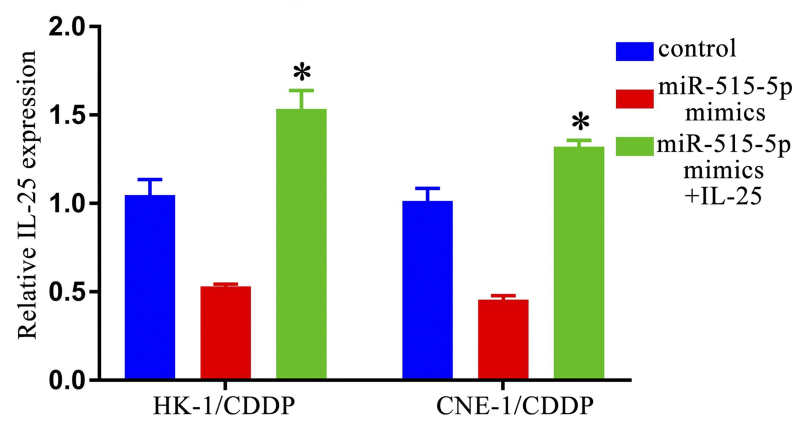

E

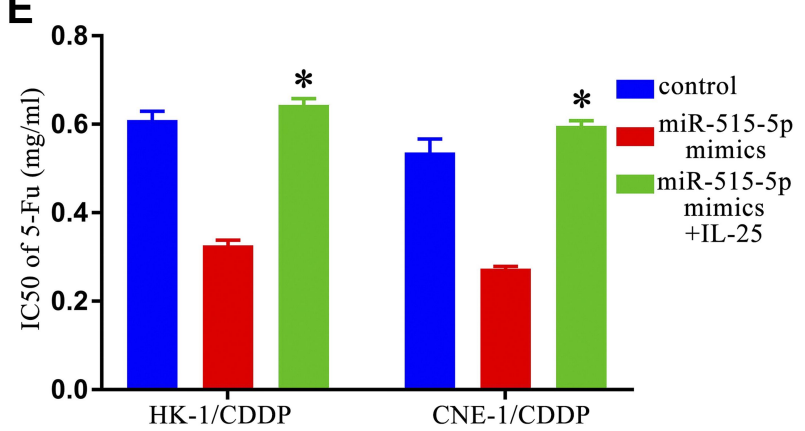

B

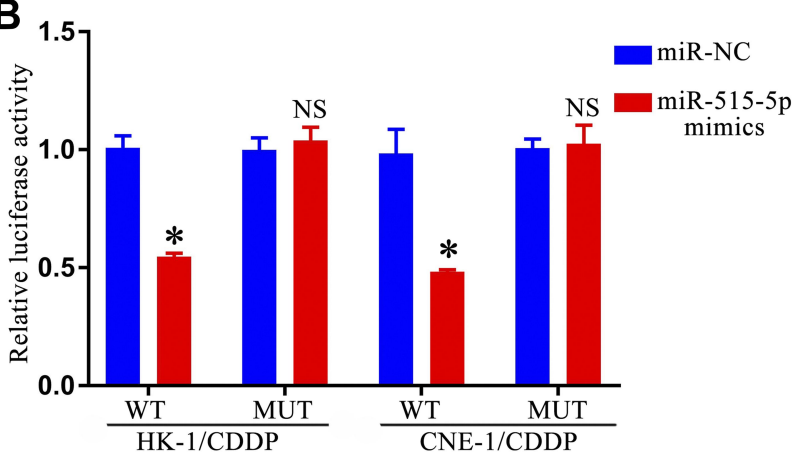

D

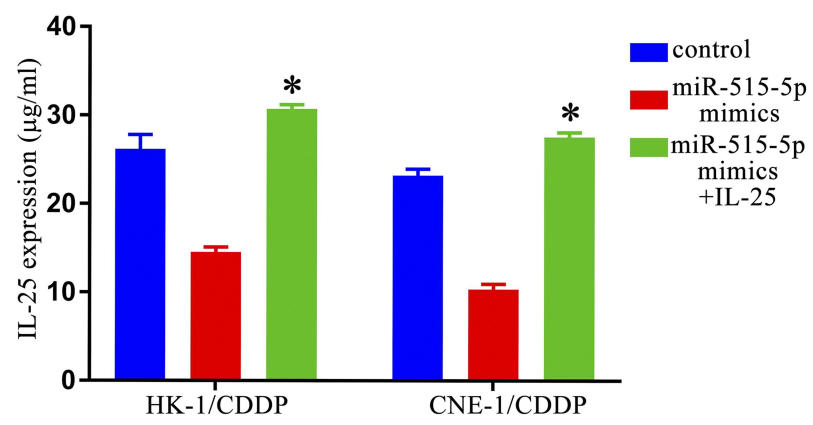

$\mathbf{F}$

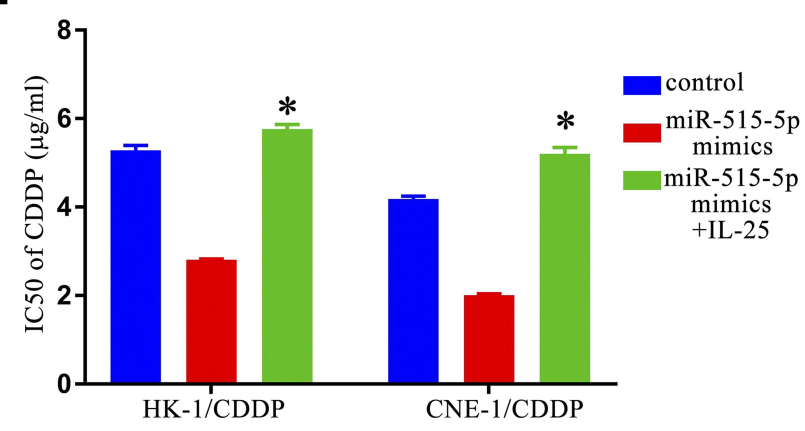

Figure 4 CircNRIPI increases NPC cell resistance to 5-Fu and cisplatin by sequestering miR-5I5-5p and thereby promoting IL-25 expression. (A). The putative miR-5I5-5p binding site in the IL-25 gene and the mutated version of this site are shown. (B). MiR-5I5-5p mimic transfection markedly impaired WT but not MUT reporter activity. (C). The expression of IL-25 was assessed via qRT-PCR (C) and ELISA (D). 5-Fu (E) and CDDP (F) IC50 values were restored in CNE-I/CDDP and HK-I/CDDP cells transfected with miR-5 I5-5p mimics following IL-25 transfection. ${ }^{N S}>0.05$, ${ }_{p}<0.05$.

prostate cancer. ${ }^{28}$ We found that the expression of miR-515$5 p$ was significantly lower in the serum of chemoresistant NPC patients, and we determined that the downregulation of this miRNA enhanced NPC cell chemoresistance through a mechanism associated with its ability to suppress the expression of IL-25. IL-25 is known to activate the NF-kB signaling pathway and to thereby increase chemoresistance in tumor cells. ${ }^{29}$ Our data provide novel evidence that IL-25 is a direct miR-515-5p target, playing an opposing role to that of miR-515-5p in regulating NPC cell 5-Fu and cisplatin resistance.

In summary, we found that circNRIP1 was upregulated in the serum of chemoresistant NPC patients. Knocking down circNRIP1 was sufficient to increase the sensitivity of NPC cells to cisplatin and 5-Fu via modulating the miR515-5p/IL-25 axis. Together these data suggest that circNRIP1 may be an important diagnostic biomarker of chemoresistance in NPC that may be amenable to therapeutic targeting in order to treat this deadly disease.

\section{Ethics Approval and Consent to Participate}

The study was approved by the Ethics Committee of Zibo Central Hospital (approval no.190603). All patients provided written informed consent prior to enrollment in the study. 


\section{Acknowledgment}

Contributed equally to this study and share first authorship: Junwu Lin and Hong Qin.

\section{Funding}

There is no funding to report.

\section{Disclosure}

The authors report no conflicts of interest for this work.

\section{References}

1. Chan AT. Nasopharyngeal carcinoma. Ann Oncol. 2010;21(Suppl 7): vii308-312. doi:10.1093/annonc/mdq277

2. Wei WI, Sham JS. Nasopharyngeal carcinoma. Lancet. 2005;365 (9476):2041-2054. doi:10.1016/S0140-6736(05)66698-6

3. Pan F, Ruan Z, Li J, et al. Radiotherapy combined docetaxel and oxaliplatin chemotherapy is effective in patients with locally advanced nasopharyngeal carcinoma. Med Oncol. 2015;32(11):252. doi:10.1007/s12032-015-0698-4

4. Colaco RJ, Betts G, Donne A, et al. Nasopharyngeal carcinoma: a retrospective review of demographics, treatment and patient outcome in a single centre. Clin Oncol. 2013;25(3):171-177. doi:10. 1016/j.clon.2012.10.006

5. He Q, Zhao L, Liu Y, et al. circ-SHKBP1 regulates the angiogenesis of U87 glioma-exposed endothelial cells through miR-544a/FOXP1 and miR-379/FOXP2 pathways. Mol Ther Nucleic Acids. 2018;10:331-348. doi:10.1016/j.omtn.2017.12.014

6. Jeck WR, Sorrentino JA, Wang K, et al. Circular RNAs are abundant, conserved, and associated with ALU repeats. RNA. 2013;19 (2):141-157. doi:10.1261/rna.035667.112

7. Chen LL, Yang L. Regulation of circRNA biogenesis. RNA Biol. 2015;12(4):381-388. doi:10.1080/15476286.2015.1020271

8. Du WW, Yang W, Liu E, Yang Z, Dhaliwal P, Yang BB. Foxo3 circular RNA retards cell cycle progression via forming ternary complexes with p21 and CDK2. Nucleic Acids Res. 2016;44 (6):2846-2858. doi:10.1093/nar/gkw027

9. Tatomer DC, Wilusz JE. An unchartered journey for ribosomes: circumnavigating circular RNAs to produce proteins. Mol Cell. 2017;66(1):1-2. doi:10.1016/j.molcel.2017.03.011

10. Zhang $\mathrm{H}$, Zhu L, Bai M, et al. Exosomal circRNA derived from gastric tumor promotes white adipose browning by targeting the miR-133/PRDM16 pathway. Int $J$ Cancer. 2019;144(10):25 01-2515. doi:10.1002/ijc.31977

11. Li XN, Wang ZJ, Ye CX, Zhao BC, Li ZL, Yang Y. RNA sequencing reveals the expression profiles of circRNA and indicates that circDDX17 acts as a tumor suppressor in colorectal cancer. J Exp Clin Cancer Res. 2018;37(1):325. doi:10.1186/ s13046-018-1006-x

12. Qiu M, Xia W, Chen R, et al. The circular RNA circPRKCI promotes tumor growth in lung adenocarcinoma. Cancer Res. 2018;78 (11):2839-2851. doi:10.1158/0008-5472.CAN-17-2808

13. Qu Y, Dou P, Hu M, Xu J, Xia W, Sun H. circRNACER mediates malignant progression of breast cancer through targeting the miR136/ MMP13 axis. Mol Med Rep. 2019;19(4):3314-3320. doi:10.3892/ mmr.2019.9965
14. Peng QS, Cheng YN, Zhang WB, Fan H, Mao QH, Xu P. circRNA_0000140 suppresses oral squamous cell carcinoma growth and metastasis by targeting miR-31 to inhibit Hippo signaling pathway. Cell Death Dis. 2020;11(2):112. doi:10.1038/s41419-0202273-y

15. Fu L, Yao T, Chen Q, Mo X, Hu Y, Guo J. Screening differential circular RNA expression profiles reveals hsa_circ_0004018 is associated with hepatocellular carcinoma. Oncotarget. 2017;8 (35):58405-58416. doi:10.18632/oncotarget.16881

16. Chen L, Zhou H, Guan Z. CircRNA_000543 knockdown sensitizes nasopharyngeal carcinoma to irradiation by targeting miR-9/plateletderived growth factor receptor B axis. Biochem Biophys Res Commun. 2019;512(4):786-792. doi:10.1016/j.bbrc.2019.03.126

17. Xie R, Tang J, Zhu X, Jiang H. Silencing of hsa_circ_0004771 inhibits proliferation and induces apoptosis in breast cancer through activation of miR- 653 by targeting ZEB2 signaling pathway. Biosci Rep. 2019;39:5. doi:10.1042/BSR20181919

18. Li X, Ma N, Zhang Y, et al. Circular RNA circNRIP1 promotes migration and invasion in cervical cancer by sponging miR-629-3p and regulating the PTP4A1/ERK1/2 pathway. Cell Death Dis. 2020;11(5):399. doi:10.1038/s41419-020-2607-9

19. Zhang X, Wang S, Wang H, et al. Circular RNA circNRIP1 acts as a microRNA-149-5p sponge to promote gastric cancer progression via the AKT1/mTOR pathway. Mol Cancer. 2019;18(1):20. doi:10.1186/ s12943-018-0935-5

20. Xu M, Jin H, Xu CX, Bi WZ, Wang Y. MiR-34c inhibits osteosarcoma metastasis and chemoresistance. Med Oncol. 2014;31(6):972. doi:10.1007/s12032-014-0972-x

21. Meng S, Zhou H, Feng Z, et al. CircRNA: functions and properties of a novel potential biomarker for cancer. Mol Cancer. 2017;16(1):94. doi:10.1186/s12943-017-0663-2

22. Hansen TB, Jensen TI, Clausen BH, et al. Natural RNA circles function as efficient microRNA sponges. Nature. 2013;495 (7441):384-388. doi:10.1038/nature11993

23. Memczak S, Jens M, Elefsinioti A, et al. Circular RNAs are a large class of animal RNAs with regulatory potency. Nature. 2013;495 (7441):333-338. doi:10.1038/nature11928

24. Zhu L, Liu Y, Yang Y, Mao XM, Yin ZD. CircRNA ZNF609 promotes growth and metastasis of nasopharyngeal carcinoma by competing with microRNA-150-5p. Eur Rev Med Pharmacol Sci. 2019;23(7):2817-2826. doi:10.26355/eurrev_201904_17558

25. Yin L, Chen J, Ma C, et al. Hsa_circ_0046263 functions as a ceRNA to promote nasopharyngeal carcinoma progression by upregulating IGFBP3. Cell Death Dis. 2020;11(7):562. doi:10.1038/s41419-02002785-3

26. Wei H, Liu D, Sun J, et al. Circular RNA circ_0008450 upregulates CXCL9 expression by targeting miR-577 to regulate cell proliferation and invasion in nasopharyngeal carcinoma. Exp Mol Pathol. 2019;110:104288. doi:10.1016/j.yexmp.2019.104288

27. Pardo OE, Castellano L, Munro CE, et al. miR-515-5p controls cancer cell migration through MARK4 regulation. EMBO Rep. 2016;17(4):570-584. doi:10.15252/embr.201540970

28. Zhang X, Zhou J, Xue D, Li Z, Liu Y, Dong L. MiR-515-5p acts as a tumor suppressor via targeting TRIP13 in prostate cancer. Int J Biol Macromol. 2019;129:227-232. doi:10.1016/j.ijbiomac.2019.01.127

29. Shen W, Qiu Y, Li J, et al. IL-25 promotes cisplatin resistance of lung cancer cells by activating NF-kappaB signaling pathway to increase of major vault protein. Cancer Med. 2019;8(7):3491-3501. doi:10. $1002 / \mathrm{cam} 4.2213$ 


\section{Publish your work in this journal}

Drug Design, Development and Therapy is an international, peerreviewed open-access journal that spans the spectrum of drug design and development through to clinical applications. Clinical outcomes, patient safety, and programs for the development and effective, safe, and sustained use of medicines are a feature of the journal, which has also been accepted for indexing on PubMed Central. The manuscript management system is completely online and includes a very quick and fair peer-review system, which is all easy to use. Visit http://www. dovepress.com/testimonials.php to read real quotes from published authors. 